\title{
Remerciements chaleureux à nos relecteurs
}

La relecture critique des manuscrits par les pairs est un mécanisme essentiel qui permet de garantir aux lecteurs une qualité élevée des publications dans le journal Radioprotection. Cette qualité s'est manifestée cette année par une augmentation très significative de notre facteur d'impact, maintenant supérieur à 1 (Bourguignon, 2021). Cette relecture critique a été assurée de façon bénévole entre octobre 2020 et décembre 2021 par des relecteurs qui acceptent de prendre le temps nécessaire dans des emplois du temps souvent très contraints. Nous les remercions chaleureusement de cette participation au travail de diffusion des connaissances dans le domaine de la radioprotection. Le succès du journal est aussi le leur.

\section{Warm thanks to our reviewers}

Critical peer review of manuscripts is an essential mechanism for ensuring readers of high quality publications in the journal Radioprotection. This quality was manifested this year by a very significant increase in our impact factor, now greater than 1 (Bourguignon, 2021). These critical evaluations were provided on a voluntary basis between October 2020 and December 2021 by reviewers who agree to take the necessary time in often very constrained schedules. We warmly thank them for their participation in the work of disseminating knowledge in the field of radiation protection. The newspaper's success is also theirs.

\author{
AMMERICH Marc, FRANCE \\ ANDRESZ Sylvain, FRANCE \\ ARBOR Nicolas, FRANCE \\ AYADI Myriam, FRANCE \\ BAGLAN Nicolas, FRANCE \\ BALOSSO Jacques, FRANCE \\ BARBOTTEAU Yves, FRANCE \\ BASSINET Céline, FRANCE \\ BEAUGELIN-SEILLER Karine, FRANCE \\ BENADJAOUD Amine, FRANCE \\ BENDERITTER Marc, FRANCE \\ BERTHO Jean-Marc, FRANCE \\ BILLARAND Yann, FRANCE \\ BOLLE Stephanie, FRANCE \\ BORDY Jean-Marc, FRANCE \\ BOURGUIGNON Michel, FRANCE \\ BREUSKIN Patrick, LUXEMBOURG \\ CESARINI Jean-Pierre, FRANCE \\ CHALLETON DE VATHAIRE Cécile, FRANCE \\ CHAPEL Alain, FRANCE \\ CHOJNACKI Eric, FRANCE \\ CONIL Emmanuelle, FRANCE \\ CORDELLE Anne, FRANCE \\ COSSET Jean-Marc, FRANCE \\ CROÜAIL Pascal, FRANCE \\ CUSSOL Daniel, FRANCE \\ DAMON Cecilia, FRANCE \\ DE CARLAN Loïc, FRANCE \\ DEVIN Patrick, FRANCE \\ DREUIL Serge, FRANCE \\ DROUET Michel, FRANCE \\ DURAND Vanessa, FRANCE
}

\author{
EBERBACH Frieder, GERMANY \\ ENOUF Olivier, FRANCE \\ ESTIER Sibylle, SUISSE \\ FARAH Jad, FRANCE \\ FEVRIER Laureline, FRANCE \\ FITTON Isabelle, FRANCE \\ FRANÇOIS Agnès, FRANCE \\ GARDAVAUD François, FRANCE \\ GAUDAIRE François, FRANCE \\ GERVAIS Philippe, FRANCE \\ GISQUET Elsa, FRANCE \\ GUERRA Rui, FRANCE \\ GREFFIER Joël, FRANCE \\ GUILLEVIC Jérôme, FRANCE \\ HABIB-GERIES Bouchra, FRANCE \\ HEMIDY Pierre-Yves, FRANCE \\ ISAMBERT Aurélie, FRANCE \\ JEFFROY François, FRANCE \\ JOLY Jérôme, FRANCE \\ JONES Kelly, ROYAUME-UNI \\ JOURDAIN Jean-René, FRANCE \\ LAFAYE Catherine, FRANCE \\ LAMA Hadid, FRANCE \\ LEBARON-JACOBS Laurence, FRANCE \\ LECLERC Elisabeth, FRANCE \\ LECOMTE Jean-François, FRANCE \\ LE GUEN Bernard, FRANCE \\ LESTAEVEL Philippe, FRANCE \\ LILAN Astrid, NORVĖGE \\ LORENTZ Sebastian, GERMANY \\ LUC Simon, FRANCE \\ MAISONOBE Jacques-Antoine, FRANCE
}


MARO Denis, FRANCE

MATHIEU Anne, FRANCE

MENETRIER Florence, FRANCE

MERCAT Catherine, FRANCE

MICHEL Celian, FRANCE

MUSEUX Emmanuel, FRANCE

NICOLAS Emmanuel, FRANCE

PASQUIER Jean-Luc, FRANCE

PERRIN Anne, FRANCE

PIRAULT Nicolas, FRANCE

POINT Sébastien, FRANCE

POURCELOT Laurent, FRANCE

RANNOU Alain, FRANCE

ROY Laurence, FRANCE

SAGE Julie, FRANCE

SAN MERCE Marta, SUISSE

SARRUT David, FRANCE

SCHIEBER Caroline, FRANCE

SCHNEIDER Thierry, FRANCE
TALBOT Jean-Noël, FRANCE

TAMPONNET Christian, FRANCE

THELLIER Sylvie, FRANCE

THOMASSIN Alain, FRANCE

TROMPIER François, FRANCE

UDOVICIC Ljiljana, ALLEMAGNE

VALETTE François, FRANCE

VAN DER MEEREN Anne, FRANCE

VECCHIOLA Sophie, FRANCE

VEYRET Bernard, FRANCE

VUILLEZ Jean-Philippe, FRANCE

ZHANG-YIN Jules Tianyu, BELGIQUE

\section{Référence}

Bourguignon M. 2021. Radioprotection : Les défis de l'open access. Radioprotection 56(3): 179-180.

Citation de l'article : 2022. Remerciements chaleureux à nos relecteurs. Radioprotection 57(1): 79-80 